\title{
RESPUESTA EDUCATIVA A LOS \\ ALUMNOS SOBRESALIENTES EN LATINOAMÉRICA
}

Luz Pérez Sánchez

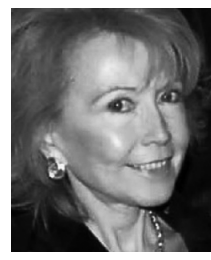

Doctora en Psicología por la Universidad Complutense de Madrid. Profesora Titular de la Universidad Complutense. Premio Nacional de Investigación Educativa. Presidenta de la Sociedad Española para el Estudio de la Superdotación. Directora del Programa para niños con altas capacidades "Programa Estrella".

Correo electrónico: [luzperezl@psi.ucm.es].

\section{RESUMEN}

Considerar las necesidades específicas de los alumnos con altas capacidades en la escuela, es una cuestión de igualdad de oportunidades respecto a los alumnos promedio y a los de baja capacidad. Esta temática sobradamente conocida desde el campo psicopedagógico, tiene un tratamiento muy diferente en distintos países y, en los últimos años, ha experimentado cambios importantes desde las políticas educativas. Este documento actualiza y efectúa un estudio sobre la situación en países de Latinoamérica y recoge información específica respecto de 
organizaciones internacionales, ministerios y administraciones de educación, universidades, asociaciones y centros específicos dedicados a la atención de este tipo de alumnado. En los países latinoamericanos, ha existido y existe un tratamiento muy desigual en cuanto a legislación e intervenciones educativas sobre este tipo de alumnado; a ello se une la falta de estudios comparativos y de conocimiento de lo realizado entre diversos países.

El análisis se establece en torno a tres grupos de variables, legislación, consideración o no de estos alumnos en el grupo de necesidades educativas especiales y tipos de medidas educativas, e investigación.

El estudio finaliza con unas conclusiones sobre la situación general y unas propuestas de mejora en los programas y políticas educativas.

Palabras clave: altas capacidades; superdotación; intervención educativa; países latinoamericanos.

\begin{abstract}
The consideration of specific needs of gifted students in the school is an issue of equality of opportunities in relation to average and low capacity students. This topic, too known from the psychopedagogical field, has been considered in a different way in several countries, and it has experienced important changes from the educational politics in the last few years. This document intends to actualise and carry out a comparative study on its situation in Latin American countries and to assemble the specific information belonging to the educational
\end{abstract}


institutions: administrations, ministries, universities, institutes, and so on, engaged in these students education There has been, and there is, a very different focus on legislation and educational interventions for this kind of students in the Latin American countries. Besides, there is a lack of comparative studies on what different countries are doing in this field.

The analysis is carried out on three variables: legislation, educational interventions and research.

The study ends with some conclusions and proposals to improve the programs and the political decisions.

Keywords : high abilities; giftedness; educational intervention; Latin American Countries.

\section{INTRODUCCIÓN}

La educación es uno de los pilares básicos de la política de progreso de un país. La mayoría de los gobiernos reconocen su importancia en el desarrollo social, pero también son conscientes de que las decisiones no son fáciles de tomar por la cantidad de variables que influyen en los procesos educativos. Nadie duda en considerar a la educación como el instrumento que mejor contribuye a aumentar la calidad de vida de los ciudadanos, así como su integración en una sociedad plural y diversa, en la que se considere como elemento de cohesión y de enriquecimiento el respeto a las diferencias.

Reflexionar sobre las necesidades específicas de los alumnos sobresalientes en la escuela es una cuestión de igualdad de oportunidades respecto a los alumnos promedio y a los de 
baja capacidad. La escuela debe afrontar la igualdad de oportunidades ante realidades diversas, puesto que sería una injusticia tratar de igual forma a niños distintos. Por tanto, la diferenciación de la enseñanza para los niños más capaces debe ser una preocupación de toda la comunidad educativa; diferenciación que debe ir dirigida a promover y apoyar el desarrollo global del niño, teniendo en cuenta todo su potencial.

Ésta es la opinión generalizada de los científicos más destacados de todo el mundo en el estudio de la superdotación y el talento. Sin embargo, se precisa una transformación en la actitud de políticos y profesionales de la educación, que implicaría un cambio de perspectiva acerca de la legitimidad de una respuesta educativa diferenciada para estos alumnos. En numerosas ocasiones, el marco legal, siguiendo los resultados de la investigación científica, es específico en su normativa en cuanto a los procedimientos de identificación y las provisiones educativas a adoptar. Sin embargo, en otras, los profesionales olvidan asumir estos procedimientos y estas medidas educativas específicas para atender a la diversidad de los más capaces, con lo que corren el grave riesgo de percibir en su escuela alumnos desmotivados, indisciplinados o con riesgo de fracaso escolar (mejor decir, fracaso de la escuela); en definitiva, toda la comunidad educativa (familias, alumnos y profesionales), estarían sufriendo las consecuencias de un programa escolar demasiado rígido como para atender a la diversidad de unos alumnos que reclaman mayor flexibilidad y adaptación curricular.

En los países latinoamericanos existió —y existe- un tratamiento muy desigual en cuanto a legislación e intervenciones educativas sobre este tipo de alumnado; a ello se une la 
falta de estudios comparativos y de conocimiento de lo que se viene realizando entre ellos. En obras ya clásicas se hace un recorrido relativamente amplio, pero revisable por el lógico paso del tiempo (Soriano, Blumen y Castellano, 2000); en otras más recientes — como las de Soriano, Fleith y Blumen (2002); Wallace y Erikson (2006) y Benavides, Maz, Castro y Blanco, (2004)— se recogen experiencias de escasos países y, además, con poca difusión entre los usuarios reales, lo cual dificulta el trasvase de conocimientos y la posibilidad de compartir documentación y experiencias. Otro dato negativo para la realización de este estudio ha sido la falta de publicaciones tanto locales como internacionales, por lo cual es difícil realizar un análisis objetivo de la situación. A pesar de todo, creemos que esta información - aunque no exhaustiva- es especialmente importante para los futuros educadores, psicólogos y pedagogos de Latinoamérica que serán los que, desde este conocimiento, potencien el futuro de la presente línea de trabajo.

Este tipo de estudios se ha realizado y difundido en 21 países de la Comunidad Europea (Mönks y Plufger, 2005), y está dando buenos frutos; prueba de ello es la formación de grupos de trabajo y proyectos comunes, como por ejemplo, el proyecto TALNET (Talnet, 2006) y otros dentro del Programa Sócrates/Conmenius, que unifican y potencian la atención educativa de los talentos en Europa (salvando las dificultades que supone, entre las naciones europeas, la diferencia de idiomas).

Para realizar este estudio - en el que pretendemos responder a esa necesidad informativa y formativa en los países de habla española—, se ha recabado información específica al 
respecto, a partir de fuentes como las organizaciones internacionales, los ministerios y las administraciones de educación de los diferentes gobiernos, las universidades, las asociaciones y los centros específicos dedicados a la atención de estos niños, de sus padres y de sus profesores, así como de fuentes bibliográficas y artículos de revistas especializadas. No obstante, es preciso señalar que la lectura y análisis de la información recogida ha estado dificultada, con frecuencia, por la escasa disponibilidad y dispersión de las fuentes bibliográficas. También queremos señalar nuevamente que este estudio no pretende ser exhaustivo, sino dar una visión actualizada de la situación en España y América Latina, que podrá ir siendo ampliada. Creemos que la mejor forma de hacerlo es presentar los datos de los distintos países situados por orden alfabético, intentando analizar su situación legislativa, sus programas educativos y su investigación específica.

\section{ARGENTINA}

En 1993, la Ley General de Educación contempla por primera vez la educación de los niños más dotados. Ninguna ley educativa había mencionado la existencia de niños con talentos académicos, ni contemplado su atención. Esta referencia se hace dentro del apartado de educación especial.

La visión que promueve la educación especial argentina es primariamente integradora. Tiene como objetivo dar respuesta tanto a niños con dificultades como a aquéllos con altas capacidades, a través de la enseñanza personalizada en el aula común, tomando en cuenta el contexto social del aula y, así, equilibrar las necesidades educativas especiales, con las del grupo, a través de las adaptaciones curriculares. En la educación 
de niños con altas capacidades, estas adaptaciones se promueven dentro del marco de la estrategia educativa de enriquecimiento.

El Acuerdo Marco para la Educación Especial, de 1998, supone un gran avance en cuanto a concientizar a la comunidad educativa de la necesidad de detectar y divulgar los materiales apropiados para la enseñanza de estos niños. En 1991, nace la Asociación de Padres de Apoyo a la Creatividad y el Talento (ACT), que fundó, en 1992, el primer colegio integrador de Argentina para los niños con talento y los niños promedio, el Instituto de Creatividad y el Talento, que funcionó desde 1993 hasta el año 2002, con un proyecto especial muy flexible para niños desde los 4 años hasta la escuela media.

El Centro para el Desarrollo del Alto Potencial (CEDAP), funciona en Buenos Aires desde el año 2000. La Universidad del CAECE ha sido la primera y la única en ofrecer un programa de posgrado para profesionales sobre superdotación y talleres de enriquecimiento para adolescentes, especialmente en las áreas de matemáticas e informática.

Existen escuelas especiales como el Colegio Norbridge (fundado en 1993) para superdotados, aunque actualmente abre sus puertas a todo tipo de alumnos. Otras escuelas incluyen programas especiales para estos niños, como el Colegio Vera Peñaloza, en Bahía Blanca. También el Centro Herman Hollerrith de Tucumán realiza programas especiales, así como el Instituto S. Bernardo Claravall, en Mendoza, y cabe citar a la Sociedad Argentina para las Ciencias Cognitivas de la Música, que promueve el desarrollo de los talentos musicales (Klosterman, 1999). 
El número de proyectos en el ámbito privado y público está creciendo; en los últimos años se han hecho investigaciones sobre creatividad e identificación del talento promovidas desde el CEDAP y recientemente se han implementado nuevos estudios, a ello ha contribuido la celebración del VI Congreso Iberoamericano de Superdotación y Talento en Mar del Plata, en junio de 2006. En este congreso se presentaron nuevas líneas de investigación sobre creatividad y aprendizaje (Saidón, 2006), alumnos talentosos en situación de riesgo (Stábile, 2006) y también una perspectiva de la situación de esta temática en el país (Vergara, 2006).

\section{BRASIL}

El interés por el superdotado se inicia en el país a partir de 1971, cuando se propuso la Ley 5.691 que fijó instrucciones y bases para la educación fundamental y media de Brasil; esta ley, en su artículo noveno, resalta la necesidad de un tratamiento especial no sólo para los alumnos con deficiencias físicas o mentales, sino también para aquellos que manifestaban una habilidad superior. Este artículo especifica que los alumnos que presentasen deficiencias físicas o mentales, los que se encontrasen en atraso considerable a la edad regular de matrícula, como los superdotados, deberían recibir tratamiento especial de acuerdo con las normas fijadas por los competentes Consejos de Educación.

Posteriormente, se han publicado numerosas disposiciones legislativas haciendo referencia a los métodos para la identificación, los programas, etcétera. En las Directrices Nacionales para la Educación Especial en la Educación Básica (Diretrizes Nacionais para a Educação Especial na Educação Básica), de 
3 de julio de 2001, se considera a los niños superdotados y con talento como dentro del grupo de educación especial y se menciona la aceleración como medida educativa para estos niños. Así mismo, se recomienda a las escuelas que organicen actividades que favorezcan el enriquecimiento de los aspectos curriculares. Sin embargo, los recursos que se destinan para desarrollar la normativa son muy reducidos. En 2002, una resolución del Ministerio de Educación sugiere la flexibilización del currículum a través de las adaptaciones, los métodos, recursos y procedimientos de evaluación.

También en el año 1971, en paralelo a la aparición de la legislación específica, se celebró el primer simposio sobre el superdotado en el país, congregando a los principales educadores interesados en el tema. Varias fueron las recomendaciones propuestas por los participantes de ese encuentro. Entre ellas se destacó la necesidad de: a) un diagnóstico precoz del superdotado; b) organización de un sistema de educación para el superdotado; c) preparación del personal especializado para atender adecuadamente las necesidades de este grupo; y d) adopción de un concepto operativo del superdotado.

En 1973 fue creado el Centro Nacional de Educación Especial (CENESP), que proporcionó un mayor apoyo a todas las iniciativas de la educación del superdotado en el país y ha patrocinado muchos encuentros de especialistas sobre el tema.

La mayoría de los programas desarrollados desde entonces son de enriquecimiento curricular, pero se realizan fuera del horario escolar regular. Organizan programas de enriquecimiento instituciones como el Centro para el Desarrollo del Potencial y el Talento, o la Secretaría de Educación del Distrito 
Federal. Existen programas especiales para niños sin recursos económicos.

También realiza actividades extraescolares, la Associação Brasileira de Superdotados, fundada en 1978 y que trata de divulgar el tema de la superdotación en Brasil. Hay dos programas para identificar y proporcionar el enriquecimiento extraescolar para los niños superdotados de Brasil: el primero tiene como principal objetivo el desarrollo emocional del niño superdotado, y el segundo busca identificar y ayudar a los niños pobres con alto potencial que, de otro modo, no serían identificados como superdotados. El Centro para el Desarrollo del Talento de Lavras (CEDET), fundado en 1993, organiza programas de desarrollo emocional para estos niños y actividades de enriquecimiento extraescolar. El municipio, Lavras, con ayuda de la Secretaría del Estado de Educación y de la Universidad Federal de Lavras, apoya financieramente la estructura general, incluyendo su personal.

El Servicio de Atención Psicoeducacional a padres de alumnos superdotados y talentosos fue creado en 2002 por el Instituto de Psicología de la Universidad de Brasilia, y tiene como objetivos: a) presentar y discutir temas relativos al superdotado, eliminando mitos respecto del mismo y sugiriendo fuentes de consulta; b) otorgar oportunidades a los padres para que compartan dudas, dificultades y éxitos respecto a la educación de sus hijos superdotados; c) discutir estrategias de estímulo del potencial del alumno superdotado en el contexto familiar, y d) concienciar a los padres sobre la necesidad de acompañar el desempeño de sus hijos superdotados en el contexto educacional. La metodología utilizada en este servicio se realiza meditante lecturas y discusión de 
textos, relatos de experiencias, dinámicas grupales, grupos de discusión, análisis de estrategias psicoeducacionales y discusión de videos.

La preocupación por los niños con talentos y con pocos recursos económicos ha estado presente en diversos programas en Brasil desde los años cuarenta, cuando la psicóloga y educadora Helena Antipoff (1892-1974) inició los programas para niños talentosos en Río de Janeiro, y continuó en Minas Gerais con grupos de niños menos favorecidos de áreas rurales.

En la actualidad se aprecia que la mayoría de los alumnos admitidos en los programas para los superdotados, en el sistema público del distrito federal, son de familias pobres y reciben poco estímulo de los padres y del ambiente. Esto también ocurre en los programas implementados en otros estados, como Pará y Espirito Santo. Por el contrario, en el Centro para el Desarrollo de Talento, los niños matriculados pertenecen a diferentes clases económicas.

En Río de Janeiro, el Instituto Rogerio Steinberg lidera un movimiento probablemente único: un programa que se dirige a los niños más pobres de la ciudad (los que viven en las favellas) y que demuestran poseer talento. El objetivo es desarrollar esos talentos y promover la creatividad.

Aunque las políticas educacionales relacionadas con la educación de superdotados fueron propuestas hace más de treinta años, proporcionalmente poca investigación sobre este tema ha sido realizada en Brasil. 
Durante dos décadas, de 1970 a 1990, la mayoría de los estudios han sido enfocados hacia la creatividad y maneras de desarrollar habilidades creativas en la clase. En la última década puede afirmarse que el foco de estudios sobre la creatividad ha variado desde el aumento de la creatividad del alumno hacia la identificación de factores que estimulan o inhiben talentos creativos en el escenario educacional. También puede observarse un número creciente de investigación en superdotados; esto resulta observable después de 1998, cuando la III Conferencia Latinoamericana sobre Superdotación se celebró en Brasil. Esta conferencia llamó la atención de educadores, psicólogos y padres sobre la importancia de atender las necesidades de los alumnos superdotados o talentosos y la implementación de programas y servicios para estos grupos. La mayor parte de las investigaciones de esta época han sido conducidas observando las percepciones de los alumnos sobre la superdotación.

Éste es el caso de los estudios de Maia-Pinto y Fleith (2002) que tuvieron como objetivo investigar la percepción de profesores, alumnos y padres sobre la actividad y estrategias educacionales implementadas en un programa específico, así como examinar la extensión en que tales actividades y las prácticas educacionales se diferencian de las utilizadas en las clases regulares. También Chagas (2003) examinó la percepción de los padres, alumnos, educadores y psicólogos acerca del fenómeno de la superdotación, y del soporte y estímulo familiar ofrecido al alumno superdotado. Los resultados indicaron que todos ellos percibían la superdotación como un conjunto de factores relacionando habilidades cognitivas y académicas superiores, y características afectivas y naturales. 
Es interesante destacar que la mayoría de las investigaciones relacionadas con la superdotación son resultado de disertaciones de tesis de maestría o de doctorado, elaboradas en programas de educación especial o de psicología escolar, y son realizadas por motivos de interés en el área.

A pesar de los esfuerzos emprendidos por los órganos gubernamentales, lo que se percibe es que los recursos destinados al área de educación de superdotados son todavía reducidos. Muchos cuestionan la necesidad de programas especiales para los alumnos talentosos con el argumento de que éstos son privilegiados y que deben ser dejados de lado a favor del alumno mediano y por debajo de la media; otros consideran de carácter elitista las propuestas educacionales que benefician a los alumnos que se destacan por un potencial superior, por ser el número de alumnos beneficiados por el programa en vigor menor que lo deseable (Soriano y Fleith, 2001).

No hay programas de graduación en educación de superdotados y talentosos en Brasil. Por lo tanto, es urgente crear un sistema de formación centrado en temas específicos relacionados con la superdotación, basados en las competencias necesarias de expertos, educadores, padres y políticos.

\section{COLOMBIA}

La Ley General de Educación (Ley 115; Congreso de la República de Colombia, 1994), que define la educación como un proceso de formación permanente, personal, cultural y social que se fundamenta en una concepción integral de la persona, de su dignidad, sus derechos y deberes, dedica su artículo 49 a "Alumnos con capacidades excepcionales". Indica 
que el gobierno facilitará en los establecimientos educativos la organización de programas para la detección temprana de los alumnos con capacidades o talentos excepcionales y los ajustes curriculares necesarios que permitan su formación integral.

Otras disposiciones adicionales mencionan la posibilidad de promocionar de forma anticipada si el alumno demuestra haber superado los objetivos de un determinado grado. Las medidas educativas se refieren a la flexibilidad curricular, los planes de estudios personalizados y graduados. A pesar de la existencia de esta normativa, los programas de atención a niños superdotados y con talentos están empezando a realizarse, y otros a planearse. Pocas instituciones públicas atienden a este colectivo.

El Instituto Alberto Merani, en Santa Fe de Bogotá, fundado en 1987, de iniciativa privada, y nivel internacional realiza cursos de formación, publicaciones y ofrecen educación específica a los niños con talento ${ }^{1}$.

Actualmente en otras poblaciones se desarrollan proyectos específicos. En la ciudad de Itagüí se lleva a cabo un proyecto para "Estudiantes con potencialidades, talentosos o capacidades excepcionales", que tiene como objetivo realizar un proceso de sensibilización, capacitación y valoración cualitativa y cuantitativa de estos alumnos, incluyendo padres y docentes de las instituciones municipales del orden estatal. Se 
pretende que el reconocimiento de la población educativa con capacidades o talento facilite el estudio de las características personales de los estudiantes, la atención oportuna de las posibles necesidades educativas especiales, la potenciación de las capacidades en cada alumno, así como la prevención del fracaso escolar a través de la asesoría familiar, la búsqueda de recursos para el mejoramiento educativo, el desarrollo social, cultural y científico de la comunidad.

En el municipio de Soacha, la Unidad de Atención Integral desarrolla un "Modelo de atención a la población excepcional y talentosa", desde el cual crea vínculos entre diferentes agentes que pueden cualificar el tipo de atención educativa para estudiantes excepcionales y talentosos. Se pretende capacitar y asesorar a los docentes para que conozcan y utilicen estrategias para la detección temprana de niños con talento. También intenta establecer convenios con instituciones de educación superior para crear una conexión entre la universidad y la educación escolar, a fin de hacer un seguimiento y apoyo adecuado de estos alumnos.

Desde el año 2000, algunos colegios de Santa Fe de Bogotá organizan también programas especiales, lo mismo que el Instituto Público Humboldt, de Barranquilla.

La realización de investigaciones y proyectos realizados en Colombia sobre este tema es relativamente escasa, esto se refleja, por ejemplo, en una mínima difusión de sus resultados. Para hacerse una idea de la situación, basta observar que en el Programa de Estudios Científicos del Instituto Colombiano para el Desarrollo de la Ciencia y la Tecnología (COLCIENCIAS, 2003), en su sección de Proyectos 
Relacionados con la Educación, no se encuentran proyectos terminados ni en curso relativos a poblaciones con superdotados ni con talentos.

El Instituto Alberto Merani, se ha destacado por su labor investigadora en diversos temas como: las necesidades de la familia del niño superdotado, la creatividad y la formación en valores (Zubiría 1998, 2006).

Un hecho destacado en el país fue la celebración del IV Congreso Iberoamericano de Superdotación y Talento, en Santa Fe de Bogotá, durante el año 2002. Este evento, que reunió a los investigadores del área, impulsó la realización de investigaciones y experiencias.

A pesar de las dificultades, van dándose pasos relevantes encaminados hacia la concientización de la sociedad sobre la importancia de atender a este sector de la población colombiana.

\section{CHILE}

El interés por este asunto comienza en los años noventa. El marco legal no considera políticas educativas relacionadas con los niños con talento, no les considera como objeto de necesidades educativas especiales, pero resalta la importancia de atender a la diversidad de todos los alumnos y recomienda la flexibilidad curricular.

La mayoría de los programas se dirigen al enriquecimiento basado en el currículum escolar y son promovidos por la Universidad Católica de Chile, desde 1993. Uno de los primeros programas comenzó en el año 1993, en la Facultad de 
Matemáticas de la Pontificia Universidad Católica de Chile; este programa piloto y pionero en la identificación y desarrollo de talentos académicos (Marshall, 2000) tuvo como objetivo detectar a niños de nueve y diez años con habilidades matemáticas, para ofrecerles una educación complementaria durante su educación escolar. Se les dieron cursos de matemáticas, especialmente diseñados por especialistas en la materia, tales como álgebra, geometría y combinatoria, algunos de ellos complementados con la utilización de un software matemático (Benavides, 2001).

A partir de esta experiencia surge el "Programa para niños con talentos académicos PENTA-UC", que funciona desde el año 2001. Busca identificar a niños y jóvenes que poseen talentos académicos sobresalientes y ofrecerles, a lo largo de una secuencia importante de años, un conjunto coherente y científicamente fundado de servicios educacionales de excelencia, que sean complementarios a las experiencias de aprendizaje que les brinda su escolaridad regular.

El objetivo central del Programa es estimular el desarrollo de las potencialidades de los niños y jóvenes talentosos a través de una formación integral, es decir, una educación que permita desarrollar el conjunto de su personalidad y no sólo sus talentos generales y específicos. Busca formar niños y jóvenes creativos, críticos y propositivos, que mantengan y aumenten su pasión por el conocimiento. Pretende, a la vez, enriquecer su proceso de maduración personal y social, facilitando el desarrollo de su autoestima, la confianza y seguridad en sí mismo y en sus capacidades, junto a una actitud ética y socialmente responsable. 
El PENTA-UC está dirigido a estudiantes que cursan entre $6^{\circ}$ año básico y $4^{\circ}$ año medio, que destacan por su potencial y habilidades académicas generales o específicas en las áreas de ciencias sociales, ciencias naturales, humanidades y/o matemáticas. El Programa ofrece a sus estudiantes un currículum variado y amplio, incluyendo cursos disciplinarios e interdisciplinarios, talleres de desarrollo personal y cursos instrumentales en inglés y computación, que los alumnos escogen de acuerdo a su motivación e intereses.

El PENTA-UC realiza una formación de enriquecimiento extracurricular compatible con la escolaridad, de aproximadamente 300 horas anuales presenciales, las que están divididas en dos períodos semestrales de 120 horas cada uno, aproximadamente. A esto se suma un período intensivo de verano, de 60 horas de duración. El Programa funciona en tres ciclos: el primero es para alumnos de $6^{\circ}$ y $7^{\circ}$ básico; el segundo es para alumnos de $8^{\circ}$ básico y $1^{\circ}$ medio; y el tercero es para alumnos de $2^{\circ}, 3^{\circ}$ y $4^{\circ}$ año medio.

En relación con actividades formativas de Chile, en 2001 se realizó el seminario internacional "Niños y jóvenes con talentos académicos: un desafío para la educación en Chile", el cual contó con la participación de especialistas internacionales. En ese mismo año se celebró el seminario para profesores "La educación de estudiantes con talentos académicos", el cual sirvió para guiar a los docentes en el proceso de identificación de talentos académicos en los niños de los colegios. También se realizó un taller para padres denominado "El rol de los padres en la educación de niños con talento", que tenía como objetivo informar a los padres en qué consiste el programa educacional en que participan sus hijos. 
Las primeras investigaciones se han desarrollado en el campo del conocimiento específico de la matemática. En el año 1998 se realizó la investigación "Talentos matemáticos: un programa en paralelo con el sistema de enseñanza en Chile", donde se describe el programa para alumnos con talento en matemáticas, desde sus orígenes, organización, objetivos y cursos realizados, los que fueron diseñados especialmente para desarrollar el talento matemático en niños y adolescentes de entre diez y dieciocho años (Benavides, 1998). Posteriormente se realiza un estudio acerca del uso de la tecnología en la educación de niños con talento, en donde se evidencia que es posible incorporar el uso de un software matemático (Maple) en un programa de enriquecimiento.

Continuando con la línea de investigaciones en un campo del conocimiento específico, como es la matemática, se está desarrollando una investigación sobre la "Caracterización de niños con talento en resolución de problemas", cuyo objetivo principal es identificar características de niños de doce y trece años con talento para apoyar su desarrollo.

En cuanto al ámbito afectivo, se está realizando la investigación "Dimensiones familiares afectivas asociadas a niños académicamente talentosos y con un autoconcepto positivo". Este estudio pretende conocer aquellas dimensiones familiares que estén simultáneamente asociadas a la condición de talento y al establecimiento de un autoconcepto positivo ${ }^{2}$.

2 Puede consultarse en: [www.puc.cl/pentauc/documentos/haz.htm]. Consultado: 27/06/06. 
La investigación "Expectativas e intereses de potenciales usuarios del programa para niños con talentos académicos (PENTA-UC): estudio cualitativo" tiene como objetivo general conocer la percepción de alumnos preseleccionados, sus padres, profesores y directores respecto a este programa, (Arancibia et al., 1998, 2000; Arancibia y García, 2006).

En otros ámbitos, el Centro está llevando a cabo el proyecto de investigación FONDEF-PENTA-UC, "Evaluación de un modelo de identificación y educación de escolares con talentos académicos, para su transferencia a organismos educacionales en Chile y Latinoamérica", que contiene varias líneas de investigación específica, orientadas al diseño y aplicación de instrumentos de medición de la calidad e impacto del trabajo desarrollado por el PENTA-UC ${ }^{3}$.

A pesar de que la política educativa no atiende de forma específica la atención a los niños talentosos, la iniciativa universitaria permite que en Chile, fundamentalmente en Santiago, la identificación y atención a este tipo de alumnado esté dando sus frutos.

\section{CUBA}

En este país, el Sistema Nacional de Educación contempla los apoyos y la atención especial a los talentos, si bien no existe una legislación explícita, está asumida por una idea general de educación personalizada. Una excepción es la normativa para la educación superior, de 1991, en la que se

Puede consultarse en: [http://www.puc.cl/pentauc] y [html/recursos/frame.htm.].

Consultado: 27/06/06. 
contempla un proceso instruccional especial para los alumnos con alto rendimiento (Reglamento de Trabajo DocenteMetodológico para la educación Superior. Resolución 269/1991, p.10). Desgraciadamente esta normativa no se extendió a otros niveles educativos. También está ausente una definición oficial de la superdotación y el talento. Sin embargo se han desarrollado diferentes modalidades de intervención, adecuadas a los intereses y necesidades del alumno, tanto desde la perspectiva del enriquecimiento con adaptaciones curriculares, currículum compacto, aceleración de cursos o asignaturas, y también existen opciones de mentorazgo para talentos específicos.

De otra parte, la atención a estos talentos también se promueve desde instituciones comunitarias, como los Centros de Cultura, los Clubs de Jóvenes Informáticos, los Centros para la Creatividad en Artes Visuales y centros igualmente específicos para jóvenes deportistas o músicos. También se promueven olimpiadas y competiciones concebidas como un modo de estímulo del talento.

La Universidad de la Habana ha tenido una larga trayectoria en los estudios sobre identificación e intervención en el talento. Resultado de esta trayectoria fue la creación, en 1997, de un departamento especializado para el desarrollo de la inteligencia, la creatividad y el talento que promueve la investigación y la intervención en estas áreas. Otras líneas de investigación han analizado tópicos referidos a la personalidad y la autorregulación de sujetos creativos.

Una época especialmente fructífera en lo relacionado con la superdotación en Cuba fueron los años ochenta; muestra 
de ello fue el proyecto ARGOS (Desarrollo de la inteligencia, la creatividad y el talento), creado con por el Ministerio de Educación con la participación de la Facultad de Educación de la Universidad de la Habana. Su objetivo era crear estrategias para atender a las necesidades educativas de los más dotados, contemplando el trabajo en las escuelas, con los padres, profesores y directivos de los centros. Una parte de este proyecto - la Comunidad Científica PAIDOS — pretendía desarrollar habilidades científicas y actitudes creativas. Desgraciadamente el proyecto ARGOS no continuó.

También se pusieron en marcha los programas PRYCREA (Proyecto para el pensamiento reflexivo y la creatividad) y TEDY (Técnicas de desarrollo intelectual), inspirados en los programas que el Ministerio de la Inteligencia de Venezuela, ponía en funcionamiento en aquellos momentos.

La atención a los alumnos superdotados y la investigación sobre esta temática ha tenido una larga trayectoria en Cuba, situación reflejada en numerosas publicaciones internacionales, si bien en la actualidad esta línea de investigación no está en un momento de expansión. No obstante, la consecuencia más importante de estos progresos ha sido la posibilidad de especialización que se ofrece a los profesores en esta materia. La mayoría de las universidades tienen cursos opcionales sobre la identificación, el desarrollo del talento y programas de enriquecimiento.

\section{ECUADOR}

Ecuador no tiene legislación específica sobre alumnos de alta capacidad. Este país fue la sede del $\mathrm{V}$ Congreso 
Iberoamericano de Superdotación y Talento, organizado por la Universidad Técnica Particular de Loja. Desde esta universidad se trabaja y proponen líneas de investigación en esa temática, algunas dirigidas a la formación de los futuros docentes (Morales, 2006). Otros grupos trabajan en cursos virtuales de formación (Zabaleta, 2006). Estas iniciativas son altamente positivas, pero en este país no existe programas generalizados de atención a este tipo de alumnado.

\section{ESPAÑ}

La legislación educativa ha ido prestando más atención a los alumnos con altas capacidades desde la promulgación, en 1990, de la LOGSE (Ley de Ordenación General del Sistema Educativo). La superdotación se menciona dentro de la categoría de alumnos con necesidades educativas especiales. El sistema educativo español propone unas líneas de actuación basadas en la aceleración y el enriquecimiento curricular, y no permite el agrupamiento en centros educativos especiales. Aunque existen importantes diferencias entre unas Comunidades Autónomas y otras, en general, se permite la aceleración o salto de curso hasta tres veces en la enseñanza obligatoria (primaria, 6-12 y secundaria 13-16 años) y uno en la no obligatoria (bachillerato 17-18). Ésta es la medida más utilizada.

La nueva Ley de Educación, publicada el 4 de mayo de 2006, incluye a estos alumnos dentro del tratamiento de "equidad en la educación" y como "alumnado con necesidades específicas de apoyo educativo", indica que "corresponde a las administraciones educativas adoptar las medidas necesarias para identificar al alumnado con altas capacidades intelectuales 
y valorar de forma temprana sus necesidades [...], y adoptar planes de actuación adecuados a dichas necesidades". Existe un procedimiento oficial para su identificación - a través de la evaluación psicopedagógica-, en la que participan especialistas, profesores y la familia. Y en todos los niveles educativos está disponible la figura del psicopedagogo u orientador (Pérez, Díaz y Domínguez, 1998).

Las experiencias en España, hoy, son numerosas; desde la década de los años 80, diferentes instituciones han ido desarrollando actividades educativas para este tipo de alumnos especialmente los denominados Programas de Enriquecimiento. El pionero, con casi dos décadas de existencia, fue el Programa Estrella de la Institución SEK, que se inició con la colaboración y asesoramiento del Departamento de Psicología Evolutiva y de la Educación de la Universidad Complutense de Madrid; éste es un programa de enriquecimiento extracurricular y apoyo psicopedagógico para los niños de alta capacidad y sus familias, que acoge alumnos de todos los niveles sociales y procedencia en edades comprendidas entre los 4 y los 16 años. También se ha llevado a cabo, de forma pionera en Europa, un "Aula de padres on-line" que forma y atiende, a través de Internet, a familias de habla española de todo el mundo. En la actualidad también existen programas de enriquecimiento en varias Comunidades Autónomas: Galicia, Madrid, Castilla-León, Navarra, etcétera (Casanova, 2002; Pérez, Díaz, y Domínguez 1998; Pérez, Domínguez, Alfaro y Escribano, 2000).

Pioneras en la investigación han sido además de la Universidad Complutense de Madrid, las de Barcelona, Santiago de Compostela, Murcia, Navarra, La Rioja y UNED. La Revista de Altas Capacidades FAISCA publica trabajos de 
investigación exclusivamente de este campo. Otras publicaciones también incluyen artículos acerca de la superdotación.

Resumiendo algunas investigaciones llevadas a cabo en universidades españolas, y que son sólo un reflejo sintético de la actividad científica actual, diremos que se inician en 1988 en la Universidad Complutense donde se realizan cinco tesis doctorales sobre intervención de distintos tipos de talentos; Prieto (1997) ha realizado varios estudios sobre evaluación e identificación del superdotado. Pérez y Domínguez, han estudiado estas características en alumnos de educación secundaria (2000), y Sánchez Manzano (2002) en niños que cursan educación primaria. Jiménez et al. (2002) describen la situación personal, familiar, social y escolar de una muestra de alumnos que han obtenido Premio Extraordinario de Bachillerato. Reyero y Touron (2003) realizaron un amplio estudio sobre la aceleración como estrategia educativa. La motivación ha sido investigada por Pomar (2001) y el talento matemático por Pasarín, Feijóo, Díaz y Rodríguez (2006). La problemática y las necesidades de las familias con hijos superdotados han sido ampliamente analizadas, así como el fracaso escolar por Pérez (2000, 2006), y la inteligencia emocional por Domínguez (2006).

Merece la pena destacar una iniciativa única en Europa: el seminario internacional sobre "Mujer y superdotación" que, desde el año 2000, organiza la Universidad Complutense de Madrid, en colaboración con la administración educativa. Anualmente se reúnen expertos internacionales que analizan la especial problemática de las niñas y las jóvenes con alta capacidad (Domínguez, Pérez, Alfaro y Reyzabal, 2003). 
El interés de la escuela pública ha ido aumentando, gracias al desarrollo legislativo y a la atención de grupos de profesionales, pero la iniciativa privada fue la primera en ocuparse de la educación de los superdotados y todavía sigue contribuyendo a su desarrollo.

\section{GUATEMALA}

Sólo recientemente se han iniciado movimientos sociales y pedagógicos a favor de los alumnos superdotados. Es destacable la labor de la Asociación Pro Alumnos Talentosos y Superdotados que, entre otras actividades, promueve la identificación de estos alumnos. La Universidad del Valle posee, desde 1992, un programa de becas y ayudas para alumnos con alta capacidad, sin recursos económicos. Recientemente (junio, 2006), el Congreso de la República, en una nueva propuesta para la Reforma de la Ley de Educación Nacional, contempla la atención a niños superdotados, aunque lo hace dentro del apartado general de educación especial: "La unidad especializada del Ministerio dictará la normativa correspondiente para la atención adecuada y no discriminatoria de la población con necesidades educativas especiales asociadas a discapacidad o no asociadas a discapacidad (problemas de aprendizaje, de comunicación, conducta) y para los niños superdotados. Todos los niños con necesidades educativas especiales, asociadas o no a discapacidad, y niños superdotados, tendrán las adecuaciones curriculares y de acceso de acuerdo a sus características para ser promovidos y certificados". Esta normativa influirá de forma clara en la implantación de programas de apoyo e intervención que, hasta ahora, no han podido ser llevados a cabo. 


\section{MÉXICO}

En 1993, la Ley General de Educación (Congreso de los Estados Unidos Mexicanos, 1993), en el capítulo IV del proceso educativo, sección 1 sobre los tipos y las modalidades de educación, precisa en su artículo 41: "La educación especial está destinada a individuos con discapacidades transitorias o definitivas, así como a aquéllos con aptitudes sobresalientes...".

No obstante, la inquietud por atender a los niños con aptitudes sobresalientes se inicia con anterioridad. En 1982, bajo la iniciativa de la Dirección General de Educación Especial (a partir de un trabajo sobre la estandarización de la escala de inteligencia Wechsler, para el nivel de primaria), identificó niños con capacidad intelectual "muy superior" en el Distrito Federal. A partir de este trabajo se promovió la realización de investigaciones con el fin de caracterizar a la población sobresaliente de edad escolar en la capital de la República. Lamentablemente, no se cuenta con los resultados de esas investigaciones.

En 1985, trece Estados de la República Mexicana (México, Quintana Roo, Querétaro, Guanajuato, Coahuila, Baja California Sur, Colima, Chihuahua, Jalisco, Tabasco, Veracruz, Chiapas y Nayarit) iniciaron la atención a niños con aptitudes sobresalientes, a través de las Direcciones de Educación Especial y a través del Programa CAS (Capacidades y Aptitudes Sobresalientes). Posteriormente, entre los años 1989 y 1994, en el marco del Programa para la Modernización Educativa, se estableció como uno de los objetivos principales para la educación especial el consolidar el Programa de Atención a Alumnos con Capacidades y Aptitudes Sobresalientes. 
El CAS se formó con niños de 5 a 12 años, dentro de la escuela ordinaria, pero donde se hallaban unas aulas llamadas CAS en las que trabajaban con maestros y profesionales especializados. También de celebran Olimpiadas del Conocimiento para los niños con talentos académicos, así como otros concursos y encuentros en los que se ponen a prueba las habilidades de los alumnos. El proyecto CAS dejó de formar parte de la atención gubernamental para convertirse en un programa de atención que permaneció sólo en los Estados antes mencionados.

Este tema quedó un tanto olvidado a raíz de la reorientación de los servicios de educación especial; sin embargo se retoma a partir del Programa Nacional de Educación 20012006, en el apartado "Política de expansión de la cobertura educativa y diversificación de la oferta", donde se señala como una de las líneas de acción: «Establecer lineamientos para la atención a niños y jóvenes con aptitudes sobresalientes".

La Secretaría de Educación Pública (SEP), desde la Dirección General de Desarrollo de la Gestión e Innovación, ha puesto en marcha un proyecto de "Investigación e innovación: una propuesta de intervención educativa para alumnos y alumnas con aptitudes sobresalientes". El proyecto se propone analizar la situación que, a nivel estatal y nacional, prevalece en relación con la atención educativa a los alumnos con aptitudes sobresalientes y construir una propuesta de intervención educativa. Se ha dividido en dos fases: una primera de diagnóstico, en 13 Estados del país; para continuar con una segunda fase en la que se desarrolla un programa de intervención. El objetivo final es crear un modelo efectivo a nivel nacional a partir de un marco conceptual que defina al 
alumno con aptitudes sobresalientes (Puga, Ramírez, Forero, Calatayud, y Santos, 2005).

Algunas propuestas diferentes, por parte de la SEP, en otros Estados son:

- En Monterrey (1999), con apoyo de la Secretaría de Educación del Estado, se prepararon maestros en la identificación y desarrollo de habilidades sobresalientes en los alumnos de escuelas públicas estatales; se trabajó con estrategias para desarrollar altos niveles de pensamiento, múltiples habilidades no académicas, mapas mentales y centros de interés portátiles.

- En Cuernavaca (Morelos), como parte del Programa de Fomento a la Innovación en la Educación Básica, avanza el Programa Niños y Niñas con Aptitudes Sobresalientes, cuyo objetivo general es promover el desarrollo intelectual, emocional y social que requieren para su formación como ciudadanos autónomos y responsables de su desarrollo personal y de la sociedad morelense, de la cual forman parte. El programa de enriquecimiento está basado en el modelo propuesto por el Proyecto Spectrum, de Howard Gardner. También existe una asociación de padres de familia de alumnos con aptitudes sobresalientes.

- En Chihuahua se ha trabajado con los modelos de Renzulli y Taylor durante diez años en preescolar, primaria y secundaria; actualmente, sólo se realiza en el nivel de primaria en la USAER-CAS. Se trabaja la creatividad y el pensamiento lógico, ya que la misión de este programa, a partir de 1996, es brindar a los niños CAS una 
amplia gama de oportunidades para que descubran y desarrollen su pensamiento creativo, habilidades de autoaprendizaje, elaboración de trabajos de investigación y creativos-productivos enfocados a la participación social.

- El Proyecto "Atmósferas creativas", surgió en 1995, en el Centro de Educación Especial y Rehabilitación (CEER), en la carrera de Psicología de la Universidad de Guadalajara, al tomar conciencia de que con el Programa CAS muchos niños con alto rendimiento intelectual no eran tomados en cuenta con la propuesta de la SEP. Además, el área emocional de dichos alumnos era poco abordada por la misma organización del proyecto. A partir de estas necesidades, y también de la reorientación de los servicios del CEER, se dio prioridad al paradigma del crecimiento y no del déficit.

Se parte de la idea de que este programa debe proponer un aprendizaje anticipador del desarrollo, en donde la realidad psicológica que el alumno construya sea mediada y anticipada por un maestro facilitador-indagador y que pase por dos planos: primero como una realidad social (diálogo con los otros); después, como una realidad psicológica (diálogo interno).

Otra idea que se defiende en este programa es que las actividades lúdicas realizadas con los alumnos, deben encaminarse a la activación de procesos cognitivos y afectivos que, de hecho existen, pero que están "dormidos". Entre los resultados obtenidos con "Atmósferas creativas" se encuentran: un incremento en la metacognición; 
motivación; autoestima; mejora de las habilidades sociales; disminución de la ansiedad; mayor creatividad, y más tolerancia a la frustración. Recientemente, esta propuesta está siendo considerada en el proyecto global que a nivel nacional se está desarrollando por parte de la SEP.

Conjuntamente con este proyecto, el Centro de Estudios e Investigación de Creatividad Aplicada organiza: un programa de enriquecimiento ("Talentitos"); un programa de verano; una escuela de padres, y coordina el Diplomado en Altas Capacidades (Valadez, Betancourt y Zabala 2006).

La Asociación Mexicana para el Apoyo a Sobresalientes (AMEXPAS A.C.), creada en 1997, ofrece servicios de identificación y asesoramiento y organiza un programa de verano; cuenta, además, con una oferta formativa para los profesionales de la educación.

En México, la investigación sobre esta temática ha evolucionado fundamentalmente por iniciativas personales, por lo que sus resultados se encuentran dispersos. Podemos destacar el trabajo de la Universidad de Guadalajara sobre instrumentos de identificación, en donde se analizó la eficacia de exámenes tradicionales y pruebas de creatividad como instrumentos de detección (Valadez, Villegas y Gutiérrez, 2002). También desde AMEXPAS se promueven programas e instrumentos de detección especialmente dirigidos a niños de comunidades indígenas. 
Recientemente se han publicado trabajos sobre el autoconcepto en niños sobresalientes (De la Torre, 2005), sobre estados emocionales y superdotación (Gomez-Pérez, 2006), y se han abierto líneas de investigación conjunta con la Universidad Complutense de Madrid, sobre la formación de padres y docentes on-line y sobre modelos para la detección de talentos ${ }^{4}$.

\section{PERÚ}

El Ministerio de Educación promueve el desarrollo de las estrategias pedagógicas para los niños superdotados y con talento en la escuela ordinaria. La Ley General de 1983 incluye, dentro de la educación especial, a ambos extremos de la diversidad educativa. En 2001, se elabora la "Guía de organización y funcionamiento de los programas de intervención con niños con necesidades educativas especiales por superdotación y/o talento". La Unidad de Educación Especial del Ministerio de Educación se ha esforzado por sensibilizar a la comunidad educativa, tanto es así que, en la nueva Ley de 2003, se contempla de forma más amplia la educación de los niños con talento; el establecimiento de programas especializados, becas y ayudas; y el considerar a los superdotados, sujetos de acción directa de la educación especial dentro del aula regular, mediante la adaptación curricular. Desde la Unidad de Educación Especial se han organizado talleres de capacitación y de sensibilización para docentes. Los avances han sido significativos en los últimos años, pero insuficientes. 
Los Programas de Fomento del Talento y la Superdotación del Ministerio de Educación del Perú tienen como finalidad promover acciones para que se observen conductas de superdotación o talento, a través del desarrollo de programas de enriquecimiento curricular en las aulas de los centros educativos piloto. Estas acciones están caracterizadas por trabajar en las aulas regulares, desde la estructura curricular vigente, con un enfoque de enriquecimiento bajo el modelo de los tres anillos propuesto por Renzulli (1986). Esta experiencia comenzó el año 2001 con siete escuelas piloto de los niveles inicial y primaria, 76 docentes y 4.000 alumnos que participaron en los distintos programas de enriquecimiento curricular. Los resultados, al término de 2001, evidenciaron la necesidad de replantear la forma de trabajo y de sentar las bases para una posterior investigación y proceso de identificación.

El año 2002 estuvo marcado por el establecimiento de centros irradiados; es decir, se replicaron los programas con las mejoras establecidas en los centros educativos piloto y se sensibilizó y capacitó a nuevos centros educativos, que fueron nominados "centros irradiados". De esta manera, cada uno de los coordinadores de los Programas de Fomento del Talento y la Superdotación, de los siete centros educativos pilotos, asesoró y acompañó la inserción de la experiencia en otros colegios cercanos a su centro piloto. La Unidad de Educación Especial del Ministerio de Educación ha utilizado como estrategia, fomentar la mejora de la calidad educativa en las aulas de los colegios participantes en el programa y sensibilizar a los centros educativos de diversas regiones del Perú sobre la necesidad de intervenir en el campo del talento y la superdotación. Para ello ha organizado numerosos talleres de capacitación para docentes y se han realizado 
visitas a las diversas regiones educativas, desarrollándose talleres de sensibilización para docentes en Piura, Cuzco, Arequipa y Puno.

Otro tipo de intervención corresponde a los programas extracurriculares, que brindan formación a los niños y jóvenes que han sido identificados como talentosos. En esta modalidad está el Programa de Atención Educativa para Niños con Facultades Talentosas y Sobresalientes (PAENFTS), de la ciudad de Lima, una experiencia que debe adecuarse a los lineamientos que establece la Unidad de Educación Especial del Ministerio de Educación y realizar investigaciones que permitan corroborar los logros que alcanzan a través del desarrollo de sus programas. La Unidad de Educación Especial del Ministerio o de Educación tiene entre sus próximos planes la creación de centros de recursos que puedan desarrollar programas extracurriculares para los alumnos talentosos y superdotados que hayan sido identificados en los centros piloto de fomento del talento y la superdotación.

Existen tres escuelas privadas para niños superdotados en Perú. La escuela Alfred Binet, en Arequipa, la escuela Leon Pinelo y la Reina del Mundo, en Lima y una organización privada, Mente Futura, en Lima, que apoya la investigación sobre la identificación y los programas para el superdotado (Blumen, 2000, 2006).

En los años 1999 y 2000 se desarrolló en Perú, y por vez primera en Latinoamérica, el programa de Especialista en Educación de Superdotados que otorga el Consejo Europeo para la Alta Capacidad (ECHA). Pero sería necesario establecer un programa de capacitación permanente para los 
profesores e incluir este tema en la formación básica de los profesionales de la educación.

La mayor parte de la investigación se promueve desde la Pontificia Universidad Católica del Perú, que trabaja conjuntamente con la Universidad Católica de Nijmegen, en Holanda.

\section{PUERTO RICO}

Los países de América Central, entre los que se hallaría Puerto Rico son, en general, quienes han tenido un mayor déficit de recursos económicos para conseguir objetivos de calidad educativa (entre los que se encontrarían una atención especializada para alumnos excepcionales).

La Organización para el Desarrollo del Pensamiento, comenzó en 1995 con programas para el desarrollo intelectual y estrategias de aprendizaje en contenidos curriculares para el desarrollo de la capacidad superior.

También en el Instituto de Investigación Psicológica de la Universidad de Costa Rica ha existido un foco de investigación en competencias intelectuales y creatividad (Tapia y Molina, 1998).

De otra parte, en la actualidad, la Universidad de Río Piedras, lidera un nuevo foco de investigación en el campo de la superdotación. Lo hace en colaboración con la Asociación de Padres de Niños Superdotados de Puerto Rico ApreNDo, creada en el año 2003. Esta asociación promovió el Primer Congreso Educativo: Niños Dotados en Puerto Rico, 
en 2004. Las conclusiones de este congreso no fueron demasiado halagüeñas: "En este país no se han desarrollado leyes que protejan los derechos de esta población. El sistema educativo no contempla cubrir las necesidades educativas de estos menores. La mayoría de los maestros y profesionales no están cualificados para atender este tipo de estudiante. Las dificultades más significativas en la actualidad se pueden clasificar en dos tipos: las educativas y las socioemocionales. Estos menores con frecuencia presentan problemas conductuales, deserción escolar y condiciones secundarias a la incapacidad para ajustarse a una realidad que los discrimina y limita su desarrollo" 5 .

\section{VENEZUELA}

La legislación general actual (Ley Orgánica de Educación, de 1986) contempla a los que tienen aptitudes superiores dentro del grupo de los que necesitan educación especial. No obstante, el primer rasgo de atención al talento en Venezuela se remonta a los años cincuenta, cuando el sacerdote jesuita Carlos Guillermo Plaza fundó el primer colegio para niños con inteligencia superior. En los años setenta, se fundaron en Venezuela una serie de organismos gubernamentales y no gubernamentales cuya labor, de alguna manera, se centraba en la identificación y la intervención de los niños con talento. El movimiento prodesarrollo de la inteligencia "Creática y de estimulación integral" es uno de los pioneros en esta década. Una de sus vertientes es conocida como "Creática educacional".

Puede consultarse en: [http://www.prgigted.org/prgoptim.htm]. Consultado: 7/07/06. 
En 1979, se establece el Ministerio para el Desarrollo de la Inteligencia que dio un gran impulso al estudio y potenciación de la inteligencia en general, desde entonces, y con distinta intensidad se vienen desarrollando diversos programas. Entre ellos se encuentra el "Programa de red de escuelas de excelencia", que fue aplicado a 44 planteles públicos venezolanos, entre 1995 y 1999. Las escuelas de excelencia son autónomas, cuentan con profesores responsables, con alumnos competentes y representantes participativos. Las aulas no son tradicionales; en lugar de pupitres, hay mesas y sillas distribuidas en círculo. El objetivo es implantar en las aulas la cultura de la inteligencia, empleando para esto debates en clase y trabajos en grupo.

En Venezuela, los movimientos de investigación sobre esta temática son puntuales y focalizados; no existen líneas que se promuevan en todo el país. En algunas universidades se han realizado tesis de grado en el área, como la de Universidad de los Andes (Mérida) por Rivera (2001), en la cual se diseñó un manual dirigido a docentes de preescolar de la Ciudad de Mérida que contribuyera a orientarlos de acuerdo con las estrategias pedagógicas que podrían utilizarse en el proceso formativo de niños con talentos superiores; la realizada por Ball e Hidalgo (2000), en la Universidad Metropolitana (Caracas), donde la detección es un objetivo central de la investigación; y la de Castañón (2000) sobre talento matemático.

Se organizan las Olimpiadas Científicas y existen también unas Olimpiadas Petroleras que involucran a los jóvenes en la actividad económica más importante del país y permite descubrir a potenciales trabajadores. 


\section{PERSPECTIVAS DE FUTURO}

En Latinoamérica, durante los años noventa, la presión ejercida por grupos de psicólogos, investigadores, profesores de todos los niveles de enseñanza y padres ha comenzado, de manera gradual y positiva, a tener sus frutos, y las legislaciones nacionales han reconocido la conveniencia y obligación de los Estados en detectar y apoyar a estos alumnos. La atención a la diversidad y la educación especial ha sido el lugar común en el que, antes o después, se han ubicado las políticas educativas de los países iberoamericanos relacionadas con las necesidades educativas de los alumnos superdotados. El nivel de regulación legislativa es diferente en los distintos países, y paradójicamente, poco coincidente con los programas de intervención que se realizan.

La sociedad reclama una educación en la que se atienda a la diversidad natural existente entre los estudiantes que puede quedar resumida en dos ideas. La primera, ha sido defendida desde que J. Dewey, a principios de siglo, pronunció su famoso discurso como presidente de la APA sobre "Psicología y práctica social”. La segunda, parte de los principios del derecho a la igualdad de oportunidades, y demanda un sistema educativo flexible para apoyar diversas alternativas de aprendizaje, permitir contenidos especiales y otros tipos de enseñanza para diferentes necesidades de los alumnos (Pérez, 1993; Beltrán y Pérez, 2000).

Las políticas educativas de los distintos países deben responder a este reto y superar políticas tradicionales, permitiendo una educación para todos según sus cualidades. Aunque ya se han dado pasos en este sentido, conviene avanzar en la consideración de los superdotados, no como unos 
privilegiados, sino como un colectivo especial que tiene sus propias necesidades educativas. Una educación de calidad no sólo pasa por disponer de recursos y tecnologías novedosas: hay que considerar diseños de enseñanza que permitan atender en las aulas talentos individuales, con necesidades educativas especiales.

Algunas conclusiones que extraemos del anterior análisis, son:

- Para realizar una adecuada detección es necesaria una mayor diferenciación y precisión conceptual entre los términos superdotado, talento, excepcional, prodigio y precoz, ya que en muchos programas educativos no quedan claramente definidos, con lo que la detección - que debe realizarse desde edades tempranas_ resulta más dificultosa.

- Existe falta de atención de los estados latinoamericanos ante su obligación legal, ética y política de capacitar a los maestros para que orienten a los niños y jóvenes de talentos y capacidades excepcionales, y para apoyar iniciativas orientadas a la detección y promoción del talento nacional.

- Aunque ha mejorado la respuesta actual, ésta no cubre las necesidades educativas especiales que demanda esta población.

- Las políticas de integración adoptadas no son una respuesta a las necesidades educativas especiales de los superdotados, y siguen implicando un desconocimiento de la complejidad y diversidad de la excepcionalidad.

- En Latinoamérica sólo existen marginalmente programas de capacitación de maestros y psicólogos para comprender 
la sobredotación intelectual, y para ofrecer programas y herramientas de formación pedagógica y didáctica requeridos para brindar la educación especial que demandan los niños y jóvenes excepcionales y talentosos.

- Para atender adecuadamente la excepcionalidad, se requieren equipos interdisciplinarios que cuenten, cuando menos, con el concurso de psicólogos y docentes especializados.

Propuestas necesarias para abordar esta situación:

- Promover un uso homogéneo e idóneo de los términos superdotado, talento, excepcional, prodigio y precoz con instrumentos válidos, confiables, prácticos y sustentados en investigaciones, unificando criterios para la identificación de la excepcionalidad.

- Fomentar, en los diversos países latinoamericanos, el abordamiento psicológico, pedagógico y didáctico de la educación de alumnos con altas capacidades intelectuales, dentro de los planes, políticas y programas educativos y formativos en general.

- Capacitar docentes, psicólogos y profesionales en las temáticas de la superdotación, la excepcionalidad y el talento en los programas universitarios de formación integral.

- Constituir redes iberoamericanas de investigadores y docentes en sus diferentes temáticas, al igual que de padres de familia, para fortalecer los programas de educación especial en las distintas regiones, estableciendo programas conjuntos de investigación, apoyados y subvencionados por organismos nacionales e internacionales. 


\section{REFERENCIAS BIBLIOGRÁFICAS}

ARANCIBIA, V., RAMÍREZ, V., HERNÁNDEZ, C., MANZI, J., LÓPEZ, A., ROSAS, R., BRALI, S., STRASSER, K., MEDINA, L., GRUEBER, V., PALACIOS, M.F. y YUS, P., Elaboración de instrumentos de medición de logros escolares de nivel cognitivo superior: Informe final., Santiago., Pontificia Universidad Católica de Chile., 1998.

ARANCIBIA, V., RAMÍREZ, V., LISSI, M.R., MANZI, J., BRALIC, S., MENDIVE, S., SEGOVIA, P. y HERNÁNDEZ, J., Evaluación diagnóstica de las habilidades socio-afectivas de los alumnos de primer año de enseñanza media de los liceos del Programa Montegrande: Informe final., Santiago., Pontificia Universidad Católica de Chile., 2000.

ARANCIBIA, V., y GARCÍA, C., Pasantías para profesores del sistema escolar regular, en un programa de educación de alumnos con talentos: resultados e impactos de su implementación., VI Congreso Iberoamericano de Superdotación, Talento y Creatividad. Mar del Plata (Argentina)., 1-3 de junio., Actas., 2006.

BAll, E., HIDAlgO, A., Propuesta de capacitación docente para detectar y estimular niños con dotación superior desde primer grado hasta cuarto grado de básica., Tesis de grado no publicada., Caracas., Universidad Metropolitana., 2000. BEltrán, J. A., y PÉREZ, L., Educar para el siglo XXI., Madrid., CCS., 2000.

BENAVIDES, M., MAZ, A., CASTRO, E., y BLANCO, C., La educación de los niños con talento en Iberoamérica., Oficina Regional de la UNESCO para América Latina y el Caribe., 
Chile., Tineo., 2004.

BENAVIDES, M., Talentos matemáticos: Un programa en paralelo con el sistema de enseñanza en Chile., Valparaíso., Tesis de Magister: Universidad Católica de Valparaíso., 1998.

BENAVIDES, M., The use of technology in a program for mathematically gifted children., Barcelona., The 14th Biennal Conference of the World Council for Gifted and Talented Children., 2001., p. 40.

BLUMEN, S., Análisis de los Programas de Enriquecimiento y Aceleración para la superdotación y el talento intelectual., VI Congreso Iberoamericano de Superdotación, Talento y Creatividad., Mar del Plata (Argentina), 1-3 de junio., Actas., 2006. BLUMEN, S., Identification of and Attention for de Highly Able in Lima., University of. Nijmegen. KUN., 2000. CASANOVA, M. A., La atención del alumnado con altas capacidades en la comunidad de Madrid., Bordón., 54 (2 y 3)., 2002., pp. 457-461.

CASTAÑón, N., Efecto del Programa Bright Start en el desarrollo de los componentes lógico-matemáticos., Madrid., Tesis Doctoral., Universidad Complutense., 2000.

CEDAP., Centro para el Desarrollo del Alto Potencial. Ministerio de Cultura y Educación de la provincia de Buenos Aires. En: [http://wwwcedalp.com]. Consultado: 7/7/06. CHAGAS, J. F., Características familiares relacionadas ao desenvolvimento de comportamentos desuperdotação em alunos de nível sócio-econômico desfavorecido., Brasilia., Dissertação de mestrado não publicada., Universidad de Brasília, D.F., 2003.

DE LA TORRE, G., "Primeros resultados de un estudio psicopedagógico sobre el autoconcepto en niños con habilidades sobresalientes"., Revista Panamericana de Pedagogía: 
Saberes y quehaceres del pedagogo., $\mathrm{n}^{\circ}$ 7., 2005., pp. 65-100. DOMÍNGUEZ, P., "Intervención educativa para el desarrollo de la inteligencia emocional"., Faisca Revista de Altas Capacidades., no 11., 2006., pp. 47-66.

DOMÍNGUEZ, P., PÉREZ, L., ALFARO, E., y REYZABAL, V., Mujer y sobredotación. Intervención escolar., Comunidad de Madrid., Dirección General de Promoción Educativa., 2003. GÓMEZ PÉREZ, M.A., Un método de terapia y de investigación en el tratamiento de familias con hijo/a con altas capacidades., VI Congreso Iberoamericano de Superdotación, Talento y Creatividad (Argentina)., 1-3 de junio., Actas., 2006. JimÉnEZ, C., ÁlvAREZ, B., AGUADO, T., GIL J. A. y JIMÉNEZ, R., Caracterización de los alumnos con premio extraordinario de bachillerato., Bordón., 54 (2 y 3)., 2002., pp. 383-398.

KLOSTERMAN, V., "Treasures of the South: Gifted and talent children in Argentina"., Gifted and Talented International., 14 (2)., $112-117$ p. 1999.

MAIA-PINTO, R. y FLEITH, D. S., "Percepção de professores sobre alunos superdotados"., Estudos de Psicologia., (19)., 2002., pp. 78-90.

MARSHALL, V., "Una experiencia piloto en la identificación y el desarrollo de talentos matemáticos", en S. BRALIC y C. ROMAGNOLI (ed.), Niños y jóvenes con talento. Una educación de calidad para todos., Santiago de Chile., Dolmen., 2002., pp. 293-298.

MÖNKS, F.J., y PLUFGER, R., Gifted Education in 21 European Countries: Inventory and Perspective., Radbound University Nijmegen., 2005. En: [wwwbmbf.de/pib/gifted_ education_21_eu_countries.pdf]., consultado: 14/06/06. 
MORAleS, G., Propuesta de la UTPL para la formación de profesores., Congreso Iberoamericano de Superdotación, Talento y Creatividad., Mar del Plata (Argentina)., 2006., 1-3 de junio., Actas.

PASARIN, M. J. FEIJOO, M., DIÁZ, O., y RODRÍGUEZ, C.E., "Evaluación del talento matemático en la Educación Secundaria"., Faisca. Revista de Altas Capacidades., no 11 ., 2006., pp. 83-102.

PEREZ, L., "Educación familiar de los niños sobredotados: necesidades y alternativas"., Sobredotaçao., Vol. 1., no $1-2$., 2000., pp. 47-64.

PEREZ, L., "Superdotación y familia"., Faisca. Revista de Altas Capacidades., no 11., 2006., pp. 17-36.

PÉREZ, L.; DÍAZ, O., y DOMÍNGUEZ, P., La educación de los más capaces: guía para educadores., Madrid., MEC., 1998. PÉREZ, L., Diez palabras clave en superdotados., Pamplona., Verbo Divino., 1993.

PÉREZ, L., DOMÍNGUEZ, P., Adolescencia y superdotación., Comunidad de Madrid. Dirección Gral. de Promoción Educativa., 2000.

PÉREZ, L., DOMÍNGUEZ, P., ALFARO, E. y ESCRIBANO, C., Educar hijos inteligentes., Madrid., CCS., 2000.

POMAR, C., La motivación de los superdotados en el contexto escolar., Santiago de Compostela., ICE., 2001.

PRIETO, M. A., Identificación; evaluación y atención a la diversidad del superdotado., Málaga., Aljibe., 1997. PUGA, I., RAMÍREZ, M.C., FORERO, A.C., CALATAYUD, A., y SANTOS, M., Proyecto de Investigación e innovación: Una propuesta de intervención educativa para alumnos y alumnas con aptitudes sobresalientes., Secretaría de Educación Básica. Dirección General de la Gestión e Innovación Educativa., México D.F., 2005. 
RENZULLI, J. S., "The three-ring conception of giftedness: A developmental model for creative productivity". En R. J. Sternberg y J.E. Davidson (eds.)., Conceptions of Giftedness., N. York., Cambridge University Press., 1986., pp. 53-92.

REYERO, M. y TOURON, J., El desarrollo del talento. La aceleración como estrategia educativa., Coruña., Netbiblo., 2003. RIVERA, M., Manual de estrategias pedagógicas dirigido a docentes de preescolar del Municipio Libertador para orientar la enseñanza de niños sobredotados., Tesis de grado no publicada., Universidad de los Andes Mérida., Venezuela., 2001.

SAIDÓN, L. M., Cómo buscarse un buen problema cruzando creatividad y aprendizaje escolar., VI Congreso Iberoamericano de Superdotación, Talento y Creatividad., Mar del Plata (Argentina)., 1-3 de junio., Actas., 2006.

SÁNCHEZ MANZANO, E., La intervención psicopedagógica en alumnos con sobredotación intelectual., Bordón., 54 (2 y 3)., 2002., pp. 297-309.

SORIANO DE ALENCAR, E. M. L., FLEITH, D. S., y BLUMEN, $S$., Trends in gifted education in South America: The Brazilian and Peruvian scenario., Gifted and Talented International, 17., 2002., pp. 7-12.

SORIANO DE ALENCAR, E. M. L., y FLEITH, D. S., Superdotados: determinantes, educação e ajustamento., São Paulo., EPU., 2001.

SORIANO DE ALENCAR, E.M.L, BLUMEN, S., y CASTELLANOS, D., "Programs and Practices for Identifying and Nurturing Giftedness and Talent in Latin American Countries". En K. HELLER, F. MÖNKS, R. STERMBERG, y R. F. SUBOTNIK. (eds.)., International Handbook of Giftedness and Talent., N. York., Pergamon Press., 2000., pp. 780-816.

STÁBILE, R. M., Educación del alumno talentoso en situa- 
ción de riesgo., VI Congreso Iberoamericano de Superdotación, Talento y Creatividad., Mar del Plata (Argentina)., 1-3 de junio., Actas., 2006.

TAPIA, N., y MOLINA, M., "Procesos cognoscitivos, aptitud y competencias académicas en la Universidad de Costa Rica"., Actualidad Psicológica., 14., 97., 1998.

TALNET., Proyecto Internacional. En: [http://www.TALNET.cz/]. Consultado: 29/07/2006.

VALADEZ, M. D., BETANCOURT, J., y ZABAL, M. A., (eds.)., Alumnos superdotados y talentosos. Identificación, evaluación e intervención. Una perspectiva para docentes., México., Manual Moderno., 2006.

VALADEZ, S. D., VILLEGAS, V., y GUTIÉRREZ, S., Identificación de niños sobresalientes en escuelas públicas de la ciudad de Guadalajara., Ponencia presentada en el IV Congreso Iberoamericano de Superdotación y Talento., Santa Fe de Bogotá (Colombia)., 9-11 de octubre., Actas., 2002. VERGARA PANZERI, M., Camino recorrido para la identifícación y atención escolar del superdotado en la República Argentina., VI Congreso Iberoamericano de Superdotación, Talento y Creatividad., Mar del Plata (Argentina)., 1-3 de junio., Actas., 2006.

WALlAnCE, B., y ERIKSON, G., Diversity in Gifted Education., London., Routledge., 2006.

ZABALETA, L.I., Curso virtual: Introducción a la superdotación., VI Congreso Iberoamericano de Superdotación, Talento y Creatividad., Mar del Plata (Argentina)., 1-3 de junio., Actas., 2006.

ZUBIRIA, M., "La familia del niño superdotado"., Psicología desde el Caribe (1)., 1998., pp. 45-56. 
ZUBIRIA, J., La formación en valores en jóvenes con talentos especiales (testimonio de dos décadas de experiencia pedagógica)., VI Congreso Iberoamericano de Superdotación, Talento y Creatividad., Mar del Plata (Argentina)., 1-3 de junio., Actas., 2006. 
